\title{
Criminologie
}

\section{L'évolution de la violence chez les adolescents québécois : phénomène et prévention}

\section{Marc Le Blanc}

Volume 32, numéro 1, printemps 1999

La justice réparatrice

URI : https://id.erudit.org/iderudit/004731ar

DOI : https://doi.org/10.7202/004731ar

Aller au sommaire du numéro

\section{Éditeur(s)}

Les Presses de l'Université de Montréal

ISSN

0316-0041 (imprimé)

1492-1367 (numérique)

Découvrir la revue

Citer cet article

Le Blanc, M. (1999). L'évolution de la violence chez les adolescents québécois : phénomène et prévention. Criminologie, 32(1), 161-194.

https://doi.org/10.7202/004731ar
Résumé de l'article

Les moyens de communication de masse nous transmettent un message alarmiste sur l'évolution de la violence, particulièrement chez les adolescents. Qu'en est-il exactement? L'analyse de la trajectoire de la violence criminelle permet de conclure que notre société vit depuis quelques années une croissance extrêmement rapide de ces délits. En particulier, les enquêtes auprès d'adolescents et d'adolescents judiciarisés signalent que ce n'est pas tant le nombre d'adolescents violents qui progresse que la fréquence de leurs actes. Il est ensuite apparu que plusieurs caractéristiques du milieu de vie des adolescents, plutôt que les facteurs macrosociaux, peuvent être responsables de cette situation : la détérioration des conditions de vie des adolescents, l'intimité accrue dans les familles, l'importance accrue des pairs et des bandes d'adolescents et leur plus grande impulsivité ainsi que la tendance à déformer la réalité. Ces facteurs ne seraient pas nécessairement responsables du niveau de la violence interpersonnelle dans notre société, ils rendraient surtout compte de sa fréquence, de sa précocité et de sa nature. En particulier, ils expliqueraient pourquoi les voies de fait sont plus fréquentes, et de loin, que les vols qualifiés alors que l'inverse était vrai à d'autres époques. Comment modifier la trajectoire à la hausse de la violence interpersonnelle? Plusieurs voies d'action préventives semblent indiquées compte tenu des facteurs qui ont été mis à jour. Ce sont l'intégration des jeunes immigrants, le développement de mécanismes de convivialité adolescente, l'amélioration du climat de vie dans les écoles, le support à la transition de l'école au milieu de travail et une intervention énergique auprès des bandes d'adolescents.
Tous droits réservés @ Les Presses de l'Université de Montréal, 1999
Ce document est protégé par la loi sur le droit d'auteur. L’utilisation des services d’Érudit (y compris la reproduction) est assujettie à sa politique d'utilisation que vous pouvez consulter en ligne.

https://apropos.erudit.org/fr/usagers/politique-dutilisation/ 


\section{L'évolution de la violence chez les adolescents québécois : phénomène et prévention}

Marc Le Blanc

Professeur titulaire

École de psychoéducation, Groupe de recherche sur les adolescents en difficulté • Université de Montréal leblancm@ere.umontreal.ca

RÉSUMÉ Les moyens de communication de masse nous transmettent un message alarmiste sur l'évolution de la violence, particulièrement chez les adolescents. Qu'en est-il exactement? L'analyse de la trajectoire de la violence criminelle permet de conclure que notre société vit depuis quelques années une croissance extrêmement rapide de ces délits. En particulier, les enquêtes auprès d'adolescents et d'adolescents judiciarisés signalent que ce n'est pas tant le nombre d'adolescents violents qui progresse que la fréquence de leurs actes. Il est ensuite apparu que plusieurs caractéristiques du milieu de vie des adolescents, plutôt que les facteurs macrosociaux, peuvent être responsables de cette situation : la détérioration des conditions de vie des adolescents, l'intimité accrue dans les familles, l'importance accrue des pairs et des bandes d'adolescents et leur plus grande impulsivité ainsi que la tendance à déformer la réalité. Ces facteurs ne seraient pas nécessairement responsables du niveau de la violence interpersonnelle dans notre société, ils rendraient surtout compte de sa fréquence, de sa précocité et de sa nature. En particulier, ils expliqueraient pourquoi les voies de fait sont plus fréquentes, et de loin, que les vols qualifiés alors que l'inverse était vrai à d'autres époques. Comment modifier la trajectoire à la hausse de la violence interpersonnelle? Plusieurs voies d'action préventives semblent indiquées compte tenu des facteurs qui ont été mis à jour. Ce sont l'intégration des jeunes immigrants, le développement de mécanismes de convivialité adolescente, l'amélioration du climat de vie dans les écoles, le support à la transition de l'école au milieu de travail et une intervention énergique auprès des bandes d'adolescents.

Criminologie, vol. 32, $n^{\circ} 1$ (1999) 
ABSTRACT The mass media tell us that violence is increasing, particularly among adolescents. What is the exact situation? We observe that there is an increase of official violent delinquency over the past decade. This observation is also confirmed for self-reported delinquency in normative and adjudicated samples. In addition, there is more of an increase in the frequency of violent acts than in participation in violent activities. This increase could be explained by macrosocial factors, but our conclusion is that it is more directly influenced by the living conditions of adolescents, family and school experiences, peer influences and changes in routine activities. We then look at possible strategies to curb this increase of violence among adolescents.

Les moyens de communication de masse nous transmettent un message alarmiste sur l'évolution de la violence, à savoir que celle-ci augmenterait énormément, particulièrement chez les adolescents. Qu'en est-il exactement? Nous tenterons de répondre à cette question à la lumière de deux types de données. Il s'agit, d'abord, des statistiques de la délinquance juvénile québécoise au cours des trente-cinq dernières années. Il s'agit, ensuite, d'enquêtes réalisées au cours des vingt dernières années auprès d'échantillons représentatifs d'adolescents et d'adolescents en difficulté de la région de Montréal.

Avant de commencer l'analyse de ces données, il convient de préciser le sens du terme «violence ». Il faut distinguer au moins cinq types d'utilisation de la force dans le cadre des relations interpersonnelles. Premièrement, il s'agit des agressions symboliques telles que mimer un sentiment de colère, claquer une porte, donner un coup de poing dans le vide, etc. Deuxièmement, il y a les agressions verbales telles que crier, répliquer, engueuler, etc. Troisièmement, il faut compter les agressions psychologiques telles que menacer, intimider, laisser craindre, etc. Quatrièmement, il faut reconnaître les agressions physiques telles qu'attaquer, se bagarrer, faire des attouchements sexuels, etc. Et, cinquièmement, il y a les agressions matérielles que constitue la destruction des biens d'autrui telle que vandaliser la propriété privée ou publique. Cet article n'aborde que deux de ces cinq types de manifestations de la violence interpersonnelle chez les adolescents, soit les agressions psychologiques et les agressions physiques. Se limiter à ces types de violence, c'est diminuer la confusion qu'entretient l'utilisation du terme générique «violence » et c'est s'intéresser à un phénomène social qui accapare, avec raison, l'atten- 
tion des citoyens, des professionnels et des organismes du système de justice et du réseau des services sociaux.

\section{La violence criminelle change d'échelle}

Il ne fait aucun doute que la quasi-totalité des sociétés occidentales ont connu une augmentation continue et substantielle de la délinquance des adolescents entre le début des années 1960 et la fin des années 1970 (Fréchette et Le Blanc, 1987 ; Cusson, 1990). Par la suite, la trajectoire de la courbe ascendante s'est stabilisée au début des années 1980 pour décroître ensuite pendant quelques années, cela avant de repartir à la hausse pour atteindre et dépasser légèrement les niveaux antérieurs les plus élevés. Cette tendance récente décrit à la fois la situation aux États-Unis (Cook et Laub, 1997) et à Montréal (Ouimet, Tremblay et Morseli, 1997). Ainsi, le taux de délinquance des adolescents de la Communauté urbaine de Montréal est passé de 41 pour 1000 à 61 pour 1000 de 1990 à 1995.

Pour leur part, les crimes contre la personne ont suivi une trajectoire récente semblable, comme le montre le figure 1 . Ainsi, les crimes contre les personnes commis par les adolescents québécois ne dépassaient pas la proportion de $10 \%$ de l'ensemble des infractions criminelles qui leur étaient attribuées jusqu'en 1987, oscillant de $6 \%$ à $10 \%$ selon les époques et avec une baisse marquée au début des années 1980 (Le Blanc, 1990a). Depuis la fin des années 1980, la proportion que représentent ces crimes augmente considérablement et, en moins de dix ans, elle a doublé, passant de moins de $10 \%$ en 1985 à un peu plus de $20 \%$ de l'ensemble des infractions criminelles des adolescents en 1995. Notons, par contre, une baisse importante en 1996. Si cette baisse se maintient en 1997 ou si la proportion des crimes avec violence se stabilise, la criminalité de violence aura changé d'échelle comme l'ensemble de la délinquance juvénile au cours des années 1960 (Fréchette et Le Blanc, 1987). La trajectoire de la délinquance avec violence est tout à fait semblable aux États-Unis. Son accroissement a débuté au même moment, c'est-à-dire en 1988, et elle est d'une ampleur tout à fait comparable jusqu'en 1995 (Blumstein, 1995 ; Snyder, Sickmund et Poe-Yamagata, 1996 ; Cook et Laub, 1997).

Cette augmentation ne s'applique pas de manière équivalente à tous les crimes contre les personnes, comme le montre la figure 2 . Au Québec, ce sont les voies de fait qui occupent une place de plus en plus 


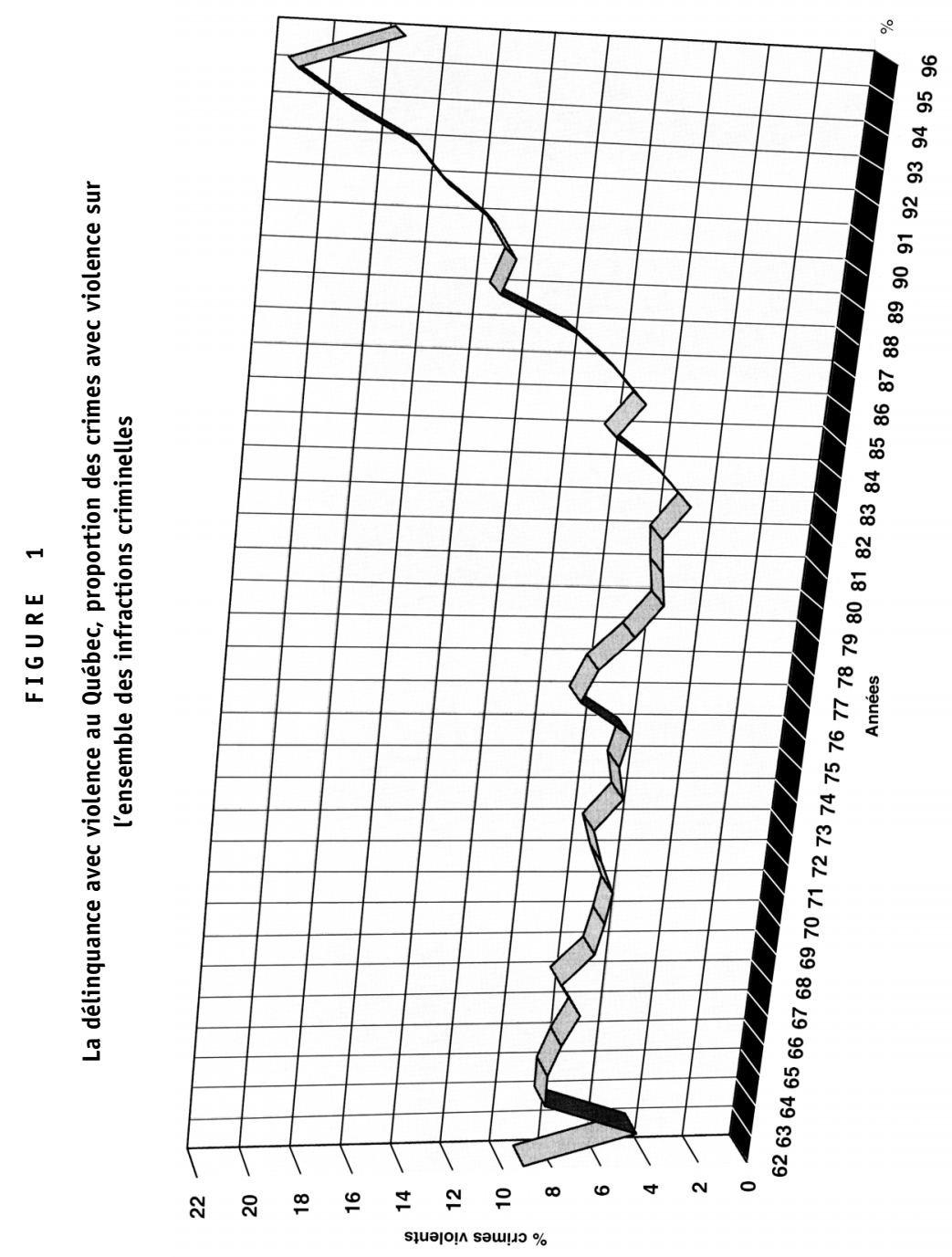




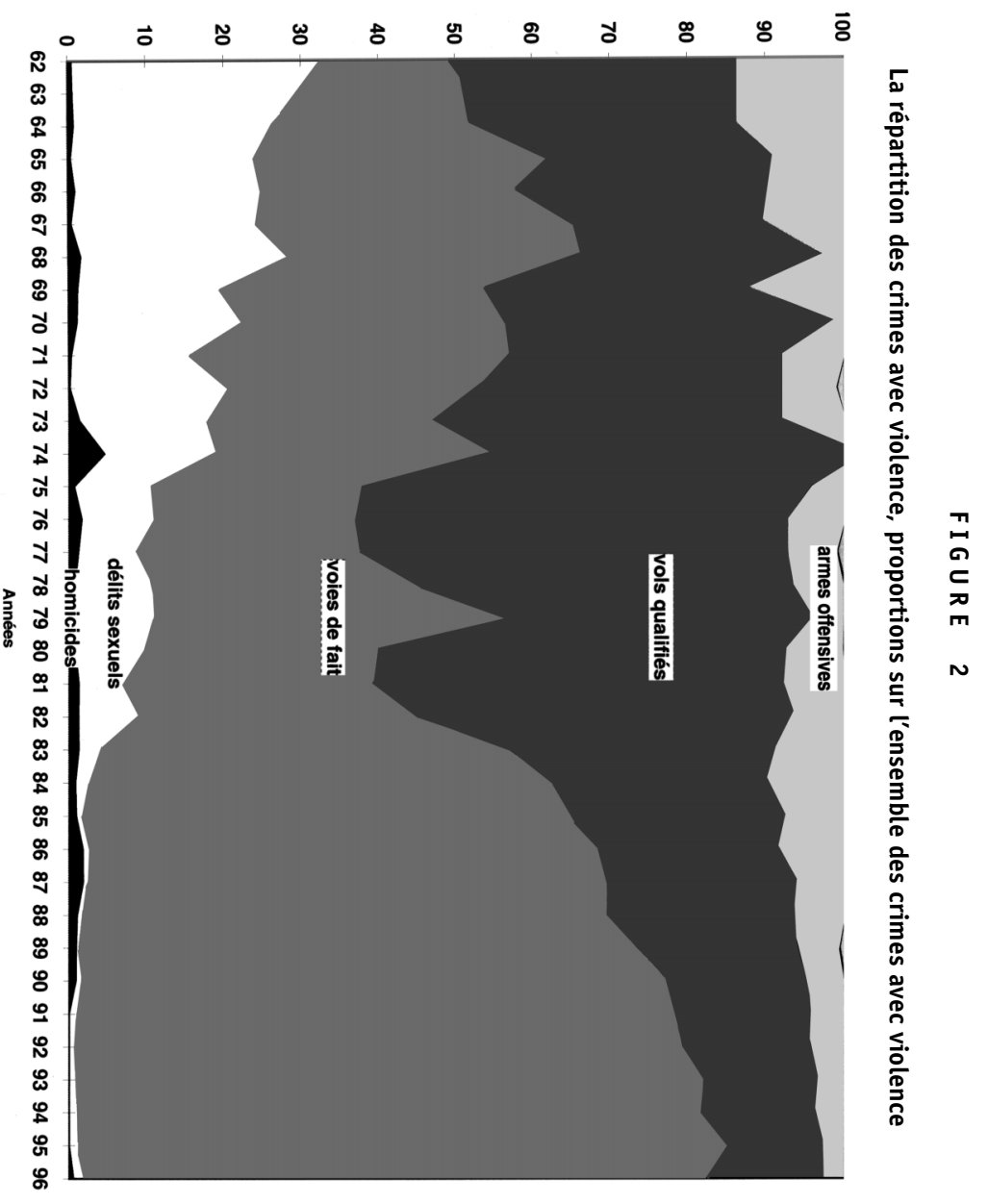


importante en comparaison des homicides et tentatives, des vols qualifiés, des agressions sexuelles et de la possession d'une arme offensive. Les voies de fait représentaient moins de $60 \%$ de l'ensemble des crimes contre la personne au début des années 1980 et elles comptaient pour $80 \%$ de ces derniers en 1995. Aux États-Unis, ce sont les homicides qui affichent la progression la plus marquée (Blumstein, 1995 ; Snyder et al., 1996 ; Cook and Laub, 1997).

Si nous analysons la distribution annuelle de ces infractions qui est représentée à la figure 2, plusieurs observations s'imposent. Une première concerne le port d'une arme offensive. Ces délits représentent une proportion minime et relativement stable des délits avec violence au Québec tout au long des 35 dernières années. Par ailleurs, ces infractions augmentent considérablement aux États-Unis (Blumstein, 1995; Snyder et al., 1996 ; Cook et Laub, 1997). Dans cette foulée, une deuxième observation s'impose. La proportion des délits sexuels apparaît comme la résultante d'une modification du Code criminel pour spécifier ces infractions au milieu des années 1980 et cette proportion avait même commencé à diminuer à partir du milieu des années 1970, au moment même où les avocats de la défense sont devenus de plus en plus nombreux devant les tribunaux pour mineurs. Par contre, ces infractions n'affichent pas de hausse depuis la fin des années 1980 ; elles demeurent à peu près au même niveau. Une troisième observation concerne les vols qualifiés et avec violence ; ces délits régressent substantiellement depuis plusieurs années. Toutefois, avec la diminution des voies de fait en 1996, ils reprennent de l'importance. Une quatrième observation a trait à l'augmentation de la proportion des voies de fait. Ces dernières progressent tout à fait proportionnellement à la diminution des délits sexuels et des vols qualifiés. En somme, la criminalité de violence des adolescents a définitivement changé de forme; elle ne concerne plus un gain sexuel ou monétaire, mais elle se manifeste principalement par l'agression d'une personne.

En somme, à long terme, les infractions avec violence des adolescents sont demeurées relativement stables pendant un quart de siècle, oscillant autour d'une moyenne de $8 \%$ de l'ensemble des délits criminels des adolescents. Par contre, depuis 1988, la proportion que représente ces délits a augmenté considérablement puisqu'elle a doublé en moins de dix ans. Nous sommes portés à penser qu' elle se stabilisera à un niveau un peu plus faible au cours des prochaines années en raison des conditions économiques qui prévalent. Comment expliquer cette progression? Avant de 
répondre à cette question, il convient de vérifier si cette progression de la violence criminelle reflète une augmentation des comportements violents rapportés par les adolescents ou une modification de la réaction sociale à la violence, soit une diminution de la tolérance de la population ou davantage de rigueur dans l'application de la loi.

\section{Les comportements violents augmentent en fréquence}

Gold et Reimer (1975) ont été les premiers à mesurer les changements de la délinquance cachée à l'aide d'échantillons d'adolescents américains au cours de deux décennies. Ils avancent une conclusion tout à fait contraire aux résultats rapportés ci-dessus pour la délinquance officielle : la fréquence et la gravité des actes délinquants des adolescents américains étaient plus faibles en 1972 qu'en 1967. Nous avons poursuivi dans cette voie avec une comparaison d'échantillons d'adolescents montréalais recrutés au milieu des années 1970 et 1980. Il ressort de cette comparaison une stabilité de la prévalence de la délinquance cachée (Le Blanc et Tremblay, 1988). En effet, $81 \%$ des adolescents qui vivaient à Montréal en 1985 rapportaient avoir commis au moins une des vingt-huit infractions au Code criminel canadien soumises par l'entremise d'un questionnaire autoadministré. La prévalence des activités illicites dans ce groupe serait inférieure à celle mesurée en 1974, alors que $84 \%$ des adolescents du même âge révélaient au moins une infraction criminelle sur une période de temps similaire. Toutefois, cette diminution de $3 \%$ n'est pas statistiquement significative. Cette évidente stabilité de la prévalence des activités délictueuses se confirme chez les garçons (prévalence de $93 \%$ en 1974 et de $90 \%$ en 1985) et chez les filles (prévalence de $77 \%$ en 1974 et de $73 \%$ en 1985). Les données des enquêtes annuelles, que rapportent Osgood, O'Mallley, Bachman et Johnston (1989) pour la période entre 1975 et 1985 aux États-Unis, confirment tout à fait les conclusions précédentes pour les adolescents montréalais.

Par contre, Le Blanc et Tremblay (1988) signalent un accroissement statistiquement significatif de la prévalence de la délinquance grave contre les biens. Osgood et al. (1989), quant à eux, notent, pour leur échantillon national d'adolescents américains, une légère augmentation des agressions contre les personnes. Cette augmentation est, par ailleurs, compensée par une faible diminution des délits contre la propriété. Ces 
données sur la délinquance rapportée par les adolescents au cours des années 1970 et 1980 concordent très bien avec l'évolution de la délinquance officielle de violence : elle n'augmente pas réellement au cours des années 1970 et 1980. Toutefois, quelques indices laissaient croire à une augmentation probable de la violence chez les adolescents montréalais. En effet, Le Blanc (1990a) rapporte l'accroissement du nombre d'adolescents montréalais qui participent à des bandes et en particulier à des bandes conflictuelles. Il observe également une augmentation du nombre d'adolescents qui sont impliqués dans des batailles entre groupes d'adolescents et qui portent une arme. Ces augmentations avaient lieu entre le milieu des années 1970 et le milieu des années 1980. Il s'agissait, après coup, de signes précurseurs de l'augmentation de la violence criminelle qui s'est manifestée au début des années 1990.

L'évolution de la délinquance autorapportée semble changer entre les années 1980 et 1990. En effet, la prévalence de la délinquance, comme de ses formes spécifiques, demeure stable, mais les fréquences des vols graves et des agressions augmentent entre les années 1970 et 1990 chez les garçons judiciarisés. Le Blanc, Girard, Kaspi, Lanctôt et Langelier (1995) rapportent des données comparatives pour quelques centaines de garçons judiciarisés en raison de troubles de comportement ou d'actes délinquants au milieu des années 1970 et en 1992. Entre ces deux échantillons, il n'y a pas de différences statistiquement significatives sur les échelles de délinquance criminelle, de délinquance grave, d'agression physique, de vol mineur et de vol grave, seul le vandalisme augmente de façon statistiquement significative. En ce qui concerne les troubles de comportement, ils sont globalement en diminution. Toutefois, la consommation des drogues illicites demeure au même niveau, alors que la promiscuité sexuelle et la rébellion familiale diminuent, tandis que la rébellion scolaire progresse. De plus, Le Blanc et al. (1995) signalent que les adolescents judiciarisés, d'alors et de maintenant, commencent leurs délits entre neuf et dix ans.

Par ailleurs, le tableau 1, adapté de Girard (1995), montre que si la prévalence de la délinquance autorapportée demeure stable chez les adolescents judiciarisés, la fréquence des vols graves et la fréquence des agressions contre les personnes augmente de manière statistiquement significative. Cela entre le milieu des années 1970 et le début des années 1990 chez les adolescents judiciarisés à Montréal.

En somme, les adolescents violents ne seraient pas plus nombreux aujourd'hui qu'hier, mais ils agiraient plus souvent aujourd'hui. C'est également ce que reflètent, en partie, les statistiques officielles sur la 
T A B LEA U 1

Adolescents judiciarisés, différences entre les années 1970 et 1990 selon les groupes d'âge

Participation

Variété

Fréquence

14-15-16 $\quad 16-17-18 \quad 14-15-16 \quad 16-17-18 \quad 14-15-16 \quad 16-17-18$

$\begin{array}{llll}\begin{array}{llll}\text { Vandalisme } & - & = & - \\ \text { Rébellion } \\ \text { familiale- }\end{array} & - & - & - \\ \text { scolaire } & & & \\ \begin{array}{l}\text { Vol mineur } \\ \text { Vol grave }\end{array} & - & = & - \\ \text { Agression } & - & = & = \\ \begin{array}{l}\text { Drogue / sexe } \\ \text { Conduite }\end{array} & + & = & = \\ \begin{array}{l}\text { Marginale } \\ \text { différence statistiquement non significative }\end{array} \\ + \text { augmentation statistiquement très significative }(\mathrm{p} \leq 0,01) \\ - \text { diminution statistiquement très significative }(\mathrm{p} \leq 0,01)\end{array}$

violence criminelle décrites ci-dessus. En effet, les statistiques policières comptent le nombre d'adolescents arrêtés indépendamment du nombre de fois qu'ils se font arrêter par la police au cours d'une année.

\section{Des changements dans les conditions de vie, les modes de socialisation et la personnalité modale des adolescents}

La violence criminelle accapare une plage élargie parmi les délits des adolescents et, de plus, les comportements violents des adolescents augmentent en fréquence sans nécessairement que la prévalence progresse. Puisque la prévalence est stable, il est difficile d'invoquer des changements sociétaux pour expliquer la progression de la violence interpersonnelle, il faut plutôt chercher l'explication de l'augmentation de la fréquence des comportements violents dans des modifications dans les modes de socialisation des adolescents.

Les changements sociétaux sont limités, sinon en opposition avec l'augmentation de la fréquence de la conduite violente des adolescents. En effet, au Québec, il n'est pas possible d'invoquer une augmentation 
de la population des adolescents, ni de l'immigration, puisqu'il s'agit de contextes stables depuis 1981 selon Ouimet et al. (1997). Il n'y a pas eu non plus de changements majeurs au Code criminel canadien depuis 1985 ni dans l'application de la loi en général. Les changements à la Loi sur les jeunes contrevenants, qui visent à rendre cette loi davantage punitive par la facilitation du transfert au tribunal pour adultes et par l'augmentation des peines pour les crimes violents, sont trop récents et ils suivent l'augmentation de la violence plutôt qu'ils ne la précèdent. Le seul changement important au Québec est l'appauvrissement de la population de Montréal et l'écart croissant entre cette région et le reste du Québec (Conseil des affaires sociales, 1989). Ces derniers changements auraient dû occasionner une augmentation des crimes contre les biens selon les connaissances criminologiques. Ce n'est pas le cas, puisque ces crimes diminuent substantiellement depuis 1991 à Montréal (Ouimet $e t$ al., 1997) et que la délinquance acquisitive demeure stable entre les années 1970 et 1990 (Le Blanc et al., 1995). Par contre, la diminution relative des crimes avec violence en 1996, en comparaison de 1995, reflète peut-être cet appauvrissement ou plutôt le désengagement des gouvernements dans les programmes sociaux.

Les chercheurs américains (Blumstein, 1995 ; Cook et Laub, 1997) suggèrent deux facteurs supplémentaires pour expliquer la progression de la violence criminelle. Il s'agit, d'abord, de la disponibilité des armes et, ensuite, des changements dans le commerce des drogues illicites, particulièrement le « crack » et la cocaïne. À Montréal, il est difficile de reconnaître à ces facteurs un rôle majeur. Premièrement, le contrôle sur les armes à feu est plus sévère qu'aux États-Unis et il se resserre avec la nouvelle loi sur l'enregistrement des armes à feu. De plus, le port d'une arme ne semble augmenter que légèrement chez les adolescents montréalais, passant de $21 \%$ en 1974 à $26 \%$ en 1985 et les adolescents ne les utilisent pas plus souvent pour se battre avec une autre personne (4\% à chaque décennie) selon Le Blanc (1990). Deuxièmement, les conflits qui résultent du commerce des drogues illicites semblent affecter davantage les motards criminalisés adultes que les adolescents, comme en font foi les journaux depuis quelques années.

La comparaison des conditions de vie et des modes de socialisation des adolescents entre le milieu des années 1970 et 1980 conduit Le Blanc et Tremblay (1988) à identifier des transformations sociales significatives dans six champs. Ces transformations expliquent probablement l'augmentation de la fréquence de la violence criminelle et des comportements 
violents des adolescents. Premièrement, sur le plan socio-économique, les adolescents des années 1980, dans leur ensemble, ont expérimenté des conditions de vie plus perturbées. D'abord, leurs parents ont vécu davantage de périodes de chômage et de dépendance de l'aide sociale et, ensuite, ils ont expérimenté moins d'aisance en raison du nombre accru de familles monoparentales. En effet, leur famille se caractérise par une mère occupant un emploi dont le prestige (et la plupart du temps la rémunération) est nettement inférieur à son niveau d'éducation. Ces conditions de vie plus difficiles s'observent également en comparant les garçons judiciarisés des années 1970 et 1990 (Le Blanc et al., 1995). En effet, 70\% de leurs familles reçoivent maintenant une forme quelconque d'aide économique contre $46 \%$ précédemment.

Deuxièmement, ces changements dans les conditions de vie sont accompagnés par des modifications importantes dans la structure de la famille au moment de l'adolescence (Le Blanc et Tremblay, 1988). Au milieu des années 1980, la famille est plus souvent monoparentale et elle est plus petite ; elle se compose d'un nombre plus restreint d'enfants. Ces changements accroissent les risques de conflits et d'escalade parce qu'il n'y a pas une tierce personne, l'autre parent ou un membre de la fratrie, pour aider la mère et l'adolescent à relativiser et à temporiser les conflits. Cette situation s'est également aggravée chez les adolescents judiciarisés au début des années 1990 en comparaison des années 1970. En effet, la proportion des familles disloquées passe de $65 \%$ à $82 \%$ au cours de ces vingt années (Le Blanc et al., 1995).

Troisièmement, sur le plan du contrôle social, non seulement le groupe familial s'est-il rétréci, mais il ressort de la comparaison des réponses fournies par les deux générations d'adolescents que les institutions les encadrent d'une façon plus serrée selon Le Blanc et Tremblay (1988). Ainsi, dans la famille, les règles de vie sont plus spécifiques et elles sont sanctionnées par un contrôle plus précis de la conduite des adolescents. Chez les adolescents judiciarisés des années 1990, les pères s'occupent moins de l'encadrement de l'adolescent, et la supervision ainsi que la discipline maternelles sont ni meilleures, ni pires (Le Blanc et al., 1995). À l'école, les adolescents participent davantage aux activités parascolaires. De plus, leurs temps libres comptent une intégration plus poussée aux loisirs organisés. En somme, il faut noter, sur le plan quantitatif, que le groupe familial est plus restreint. Et il faut retenir que l'encadrement est renforcé. En conséquence, les adolescents des années 1980 recevaient des pressions socialisatrices plus contraignantes dans la 
famille, à l'école et dans leurs autres milieux de vie, ce qui n'était toutefois pas le cas pour les adolescents judiciarisés.

Quatrièmement, sur le plan de la convivialité, la contrepartie de l'encadrement plus rigoureux des adolescents, c'est l'élargissement de leur réseau de pairs. Les pairs n'ont pas une importance affective plus grande qu'avant, mais ils sont plus nombreux et ils font l'objet de relations plus intensives. Sebald (1989) observe également que les adolescents d'aujourd'hui demandent plus qu'antérieurement l'avis de leurs pairs sur diverses questions. De plus, Le Blanc (1990a) signale la réapparition cyclique des bandes conflictuelles et il note qu'elles sont présentes à Montréal à la fin des années 1980. Toutefois, les adolescents judiciarisés des années 1990, en comparaison de ceux des années 1970, ne participent pas davantage aux bandes marginales (Le Blanc et Lanctôt, 1995). Environ les deux tiers le font aux deux époques. Toutefois, même s'il est clairement démontré que les bandes recrutent les individus les plus marginaux parmi les adolescents (Thornberry et al., 1993 ; Lanctôt et Le Blanc, 1996), il est également établi que la participation à une bande est passagère, d'une durée d'environ une année, mais qu'elle accroît les comportements violents pendant cette période (Thornberry, 1997 ; Le Blanc et Lanctôt, 1997).

Cinquièmement, sur le plan scolaire, la comparaison des données sur l'adolescent à l'école montre, selon Le Blanc et Tremblay (1988), que les adolescents d'aujourd'hui croient davantage que l'école est un bon moyen de promotion sociale. Malgré cette croyance, ils ne sont pas plus assidus qu'avant dans leurs tâches scolaires et le succès scolaire n'est pas une valeur plus significative pour eux. Chez les adolescents judiciarisés, Le Blanc et al. (1995) montrent que leurs aspirations scolaires augmentent, mais qu'ils ne réussissent pas davantage. Toutefois, le décrochage scolaire n'augmente pas pour autant.

Sixièmement, Le Blanc et al. (1995) montrent qu'il y a maintenant une proportion importante des adolescents judiciarisés dont les parents sont nés à l'extérieur du Canada, $31 \%$, ou qui sont eux-mêmes nés à l'étranger, $19 \%$. De plus, la proportion des membres des minorités visibles est maintenant substantielle parmi les pupilles de la Chambre de la Jeunesse, ce qui était exceptionnel au début des années 1970.

Non seulement observe-t-on des changements dans les conditions de vie et les modes de socialisation des adolescents, mais des transformations de la personnalité modale des adolescents peuvent également rendre compte de l'augmentation de la violence criminelle et de l'accroissement 
de la fréquence des comportements violents. Utilisant treize échelles des questionnaires de personnalité de Jesness et d'Eysenck, nos travaux (Le Blanc et Côté, 1986 ; Le Blanc et Tremblay, 1988) ont décrit des modifications significatives sur moins de la moitié d'entre elles. Les différences les plus marquantes entre la personnalité modale des adolescents des années 1970 et celle des années 1980 concernent la progression de la méfiance, de la perception de sentiments personnels plus agressifs et d'une conscience plus aiguë des sentiments qui animent l'adolescent. Des variations secondaires s'observent au niveau de la capacité de reconnaître les éléments déplaisants de la réalité, de l'augmentation de l'émotivité et de l'insécurité, sans oublier une plus grande tendance à l'isolement.

S'il n'y a pas plus d'adolescents, au milieu des années 1980, qui apparaissent inadaptés, c'est-à-dire qui présentent des résultats au-dessus des normes des inventaires de personnalité, il n'en demeure pas moins que la personnalité modale a été altérée par les transformations sociales. Même si les adolescents des années 1980 apparaissent comme relativement décentrés, confiants face aux autres, plutôt réalistes face aux difficultés qu'ils rencontrent dans leurs relations interpersonnelles et même si leurs résultats traduisent un bon niveau de socialisation, ils semblent néanmoins plus anxieux et plus insécures en comparaison des adolescents du milieu des années 1970. Cette insécurité se manifeste par une plus grande émotivité ; elle s'accompagne également d'une agressivité plus marquée. En ce sens, nous pourrions dire que leur crise d'identité, leur crise d'adolescence, s'affiche. selon des modalités quelque peu différentes au milieu des années 1980 en comparaison de la crise d'adolescence au cours de la décennie précédente. Deux études récentes supportent cette conclusion. Il s'agit des données rapportées par Pancoast et Archer (1988), qui portent sur les échelles de personnalité du MMPI à travers quatre décennies, et de celles qui sont publiées par Bovasso et al. (1991) ; elles concernent les valeurs morales selon l'échelle de Crissman.

Entre les années 1970 et 1990, Le Blanc et al. (1995) notent moins de différence significative chez les adolescents judiciarisés que chez les adolescents des années 1970 et 1980 sur les inventaires de personnalité de Jesness et de Eysenck. De fait, une seule échelle distingue clairement les deux groupes d'adolescents judiciarisés, il s'agit de l'autisme. Les adolescents judiciarisés d'aujourd'hui manifestent une propension, dans la pensée et la perception, à déformer la réalité selon leurs propres désirs et leurs propres besoins. Par ailleurs, sur trois échelles, il y a une tendance à ce que les adolescents judiciarisés d'aujourd'hui accentuent des caractéris- 
tiques qui les démarquent habituellement des adolescents conventionnels. Ils ont davantage de difficultés à rencontrer les exigences sociales par des voies socialement approuvées ; ils sont orientés davantage vers le monde criminel ; ils partagent encore plus l'éthique du dur et le désir prématuré du statut d'adulte ; ils manquent encore plus de satisfaction de soi ; et ils ne sont pas davantage passifs et déprimés.

Ainsi, il faut retenir que, d'une part, il y a chez les adolescents une solidification de l'allocentrisme, tandis que, d'autre part, chez les adolescents judiciarisés, il y a une consolidation de l'égocentrisme. Pour les premiers, il s'agit d'un renforcement de l'obligation morale puisque, malgré une accentuation de leur instabilité émotionnelle et de leur impulsivité, il faut noter qu'ils sont plus réalistes et plus conscients des sentiments qu'ils provoquent. Tandis que, pour les seconds, il s'agit d'une détérioration de l'obligation morale qui se manifeste par une construction fantaisiste de la réalité et par davantage d'asociabilité.

En somme, qu'il s'agisse des changements dans les conditions de vie, les modes de socialisation ou la personnalité modale, les tendances observées chez les adolescents des années 1970 en comparaison de ceux des années 1980 se poursuivent vraisemblablement au cours des années 1990. En effet, elles se manifestent également en comparant les adolescents judiciarisés des années 1970 à ceux des années 1990. Il est donc raisonnable de conclure que ces changements expliquent la stabilité de la prévalence des conduites violentes et l'augmentation de leur fréquence chez les individus violents.

\section{Prévention de la violence interpersonnelle}

L'analyse de l'évolution de la violence criminelle a permis de conclure que notre société vivait depuis quelques années une croissance extrêmement rapide de ces délits. En particulier, les enquêtes auprès d'adolescents et d'adolescents judiciarisés signalent que ce n'est pas tant le nombre d'adolescents violents qui progresse mais plutôt la fréquence de leurs actes et le rajeunissement des personnes violentes. Il est ensuite apparu que plusieurs caractéristiques du milieu des adolescents, plutôt que les facteurs macrosociaux, pouvaient être responsables de cette situation : la détérioration des conditions de vie des adolescents, l'intimité accrue dans les familles, l'importance des pairs et la réapparition des bandes d'adolescents et leur plus grande impulsivité et capacité de déformer la réalité selon leurs 
besoins. Ces facteurs ne seraient pas nécessairement responsables du niveau de la violence interpersonnelle dans notre société, ils rendraient surtout compte de sa fréquence, de sa précocité et de sa nature. En particulier, ils expliqueraient pourquoi les voies de fait dominent actuellement, et de loin, les vols qualifiés alors que l'inverse était vrai à d'autres époques.

Par ailleurs, Le Blanc (1997) a conclu que l'analyse du cheminement de l'individu qui en vient à commettre des infractions avec violence démontre qu'il s'agit d'une activité de la fin de l'adolescence et de la jeunesse, dont la répétitivité et la durée sont faibles, et qui a habituellement comme précurseur d'autres activités délictueuses. Il a été établi que la stabilité et la continuité à travers le temps de ce type de conduite sont grandes, entre les gestes d'agressivité au début de l'enfance, les bagarres au moment de l'adolescence et les délits avec violence à l'âge adulte. Par ailleurs, la violence psychologique et physique s'ajoute progressivement dans le répertoire des conduites marginales des individus, mais elle n'a pas un développement autonome. Il a finalement été établi qu'il est possible de prédire l'apparition de ce type de conduite dans la mesure où sont respectées trois conditions : utiliser prioritairement des marqueurs comportementaux, ne pas remonter trop loin dans le passé de l'individu et compléter les prédicteurs comportementaux avec des variables précises relatives à l'intégration sociale, aux caractéristiques de la personne, aux caractéristiques du quartier et à des situations spécifiques qui sont génératrices de violence interpersonnelle.

Ces deux niveaux d'analyse de la violence interpersonnelle sont complémentaires et ils appellent des stratégies de prévention interdépendantes. Complémentaires parce que les transformations dans les modalités de socialisation des adolescents sont autant de contextes qui peuvent favoriser l'agir violent chez les individus qui sont plus susceptibles que d'autres d'adopter de telles conduites. Cette complémentarité implique aussi un plan global de prévention de la violence interpersonnelle. Global parce que concerné par ces deux niveaux d'action. Nous ne traiterons pas ici de la prévention générale de la délinquance par des interventions défensives et de développement social comme nous l'avons fait ailleurs (Fréchette et Le Blanc, 1987 ; Vitaro, Gagnon, Dobkin, Le Blanc, 1994 ; Le Blanc, 1995). Nous nous intéressons plutôt à la prévention spécifique, c'est-àdire aux actions qui adoptent une cible limitée, soit la violence interpersonnelle chez les adolescents.

Cette prévention spécifique implique des actions qui concernent directement des groupes et des individus susceptibles de produire des gestes qui 
comportent de la violence psychologique et physique de nature criminelle. En adoptant la perspective de la prévention spécifique de la conduite violente, nous ne traiterons pas des programmes de prévention qui sont efficaces pour atténuer les facteurs de risque de la conduite violente puisque plusieurs recensions inventorient ces programmes (par exemple Hawkins, Catalano et Brewer, 1995 ; Yoshikawa, 1995). De plus, nous nous référerons à la littérature nord-américaine parce qu'on y trouve des programmes qui ont été évalués rigoureusement et que l'évolution de la violence interpersonnelle chez les adolescents suit la même tendance partout en Amérique du Nord. Il existe des programmes québécois de prévention de la violence, mais nous nous limiterons à ceux ayant été évalués systématiquement.

\section{Actions sur les groupes}

Comment altérer la tendance ascendante de la violence interpersonnelle? D'abord, il faut reconnaitre précisément la nature de la violence psychologique et physique qu'il convient de prévenir. Compte tenu que ce sont les voies de fait et les batailles entre groupes d'adolescents plutôt que les vols qualifiés qu'il s'agit avant tout de réduire, les interventions défensives seront fort probablement peu utiles. Il ne s'agit pas d'augmenter la surveillance et de faire des aménagements physiques pour réduire les infractions, comme dans le cas des vols à main armée dans les banques au cours des années 1970 (Gabor, Baril, Cusson, Élie, Le Blanc et Normandeau, 1987). Une surveillance policière accrue dans les transports publics et des aménagements physiques (meilleure illumination des couloirs, caméras...) fera peut-être diminuer les agressions dans ces lieux, mais il est aussi fort probable que celles-ci se déplaceront vers d'autres lieux comme la diminution des vols de banques à Montréal s'est accompagnée d'un accroissement phénoménal des vols chez les dépanneurs.

Cinq voies d'action préventive semblent particulièrement indiquées par les facteurs qui ont été mis à jour pour orienter une stratégie globale d'intervention contre les voies de fait et les batailles entre groupes d'adolescents. Ce sont l'intégration des jeunes immigrants, le développement des mécanismes de convivialité adolescente, l'amélioration du climat de vie dans les écoles polyvalentes, le support à la transition de l'école au milieu de travail et une intervention énergique sur les bandes d'adolescents. D'autres interventions visant l'école, la famille, les désavantages socio-économiques et l'habitation sont envisageables, mais elles relèvent du développement social général, donc de la prévention générale de la 
délinquance et non de la prévention du phénomène spécifique de la violence interpersonnelle. Même pour les voies d'intervention que nous discuterons, nous n'avons pas la prétention d'être exhaustif en termes d'interventions possibles (pour une description plus exhaustive des programmes intéressants, voir Brewer et al., 1995).

Intégrer les adolescents de l'immigration

Les adolescents dont les parents sont des immigrants de première ou de deuxième génération, pour des raisons légitimes (se retrouver entre personnes qui partagent une même langue, une même culture, etc.) ou par l'impact de mécanismes d'exclusion (racisme, ghetto, etc.), tendent à se rassembler en groupes ou bandes culturellement homogènes (Le Blanc, 1991). Ils deviennent ainsi hautement visibles et menaçants pour la majorité et, naturellement, ils sont alors exclus des activités organisées pour les adolescents (activités sportives, culturelles, de convivialité). Il s'ensuit que des programmes d'intégration de ces adolescents aux activités de la majorité sont essentiels. Il faut que les services sportifs et de loisirs, les associations diverses et les maisons de jeunes fassent un effort supplémentaire pour se rendre accessibles et qu'ils aillent recruter ces adolescents de familles immigrantes pour faciliter leur intégration à l'ensemble de la société.

\section{Favoriser la convivialité adolescente}

Précédemment, nous avons mentionné que les adolescents d'aujourd'hui étaient moins individualistes et qu'ils recherchaient davantage la présence de leurs pairs, ceci, entre autres, parce que la famille devient de plus en plus restreinte et contrôlante. Une autre voie pour la prévention de la violence interpersonnelle s'impose à la suite de cette observation : augmenter les opportunités pour que s'exerce la convivialité adolescente sous la supervision d'adultes. Maisons de jeunes, mouvements de jeunesse, groupes sociaux, etc. sont autant de moyens de favoriser les interactions entre adolescents, et ce, surtout dans un contexte où les adultes ne sont pas absents et où l'on met l'accent sur le développement de la personne (Felson (1994) développe cette voie). La meilleure compréhension qui résultera de l'intégration des adolescents de familles d'immigrants à la vie sociale de l'ensemble des adolescents, et qui sera aussi accompagnée par une convivialité renouvelée entre adolescents, constituera un gage de la prévention des conflits interpersonnels. 
Réduire la violence dans les écoles

Il y a de multiples façons de réduire la violence à l'école au moment de l'adolescence, qu'il s'agisse de modifier le programme scolaire, les activités parascolaires, l'environnement physique, l'organisation de l'école, les opportunités de violence (détecteur de métal, déplacement des chefs de gangs, entre autres), etc. (voir Côté, Le Blanc, Ouellet et Pronovost, 1988 ; Goldstein, 1992). Deux catégories de programmes ont été adéquatement évaluées et ils donnent des résultats significatifs.

D'abord, il y a des programmes conçus pour réduire la brutalité de certains élèves à l'égard des plus jeunes et des plus faibles. Olweus (1991) propose un programme de ce type qui comprend les composantes suivantes : un dépliant pour informer les professeurs sur le phénomène et les moyens d'y faire face, un dépliant similaire pour les familles, une cassette vidéo présentant des situations de brutalité de la part d'adolescents, un questionnaire pour faire l'inventaire de la situation et une session de formation pour la direction de l'école et ses professeurs. Ce programme a diminué la brutalité dans les écoles de Norvège de $50 \%$.

Ensuite, il y a des programmes pour modifier le fonctionnement de l'école. Qu'il s'agisse de PATHE (Positive Action Through Holistic Action) de Gottfredson (1986), de CARE de Gottfredson (1987) ou du Multicomponent School Organization Intervention (Gottfredson et al., $1989,1993,1996)$, ces programmes comprennent plusieurs des composantes suivantes : un comité de divers partenaires pour la conception, la planification et l'implantation du programme ; une révision de la politique disciplinaire ; des innovations au niveau de l'enseignement (enseignement coopératif, développement d'habiletés) ; amélioration de la gestion de classe (proactive, approche de réalité) ; amélioration du climat de l'école par des activités parascolaires et une campagne d'estime de l'école ; des programmes pour faciliter la transition de l'école au marché du travail ; des services spécialisés ou une approche comportementale. La combinaison de plusieurs de ces composantes diminue les suspensions, les sanctions de la part des autorités scolaires, la consommation des drogues, la délinquance sous toutes ses formes et elle augmente l'ordre et l'organisation dans les classes ainsi que l'attention des élèves.

\section{Accélérer la transition de l'école au milieu du travail}

La transition de l'école au marché du travail est plus difficile pour les adolescents qui ne terminent pas leur secondaire (Le Blanc et Pronovost, 
1979, 1980 ; Le Blanc, Janosz et Langelier-Biron, 1993). C'est aussi une source importante de délinquance (voir Le Blanc, Vallière et McDuff, 1992, 1993). Si, par un effort conjoint des milieux scolaires (programmes qui combinent le travail et les études, programmes scolaires adaptés, etc.) et des entreprises (réserver un certain nombre d'emplois pour des jeunes), on pouvait intégrer plus de jeunes au marché du travail, il y aurait moins d'adolescents inoccupés. Leur insertion sociale serait, comme il est bien établi, un frein puissant à l'activité délictueuse. Les programmes de support pour l'obtention de diplôme, les « Quantum Opportunity Programs» de la Fondation Ford par exemple, semblent particulièrement efficaces puisqu' ils peuvent réduire la délinquance grave d'environ 50 \% (Greenwood, Model, Rydell et Chiesa, 1996). Ces programmes cherchent à garder les adolescents à l'école par des récompenses financières immédiates ou futures qui sont associées aux résultats scolaires.

\section{Réduire l'influence des bandes marginales}

Une autre voie, c'est l'intervention sur les bandes d'adolescents, surtout les bandes conflictuelles. Ces programmes prennent quatre formes : l'organisation communautaire, le travailleur de rue, la suppression des bandes et l'intervention de crise. Selon Howell (1997) aucune de ces stratégies n'a réduit de façon satisfaisante la violence. Le programme proposé et évalué par Spergel (1995) réduit de moitié l'augmentation de la violence ainsi que d'autres formes de délinquance. Il s'agit de combiner la mobilisation de la communauté, les actions de suppression (rendre les bandes moins attrayantes pour les membres périphériques en les surveillant et en les harcelant, neutraliser le leader et les membres du noyau central, rendre les batailles plus difficiles par la surveillance en utilisant des informateurs, etc.) et les interventions sociales et de développement des opportunités d'intégration sociale (formation, emploi, support familial, etc.).

À ces actions qui ciblent directement les bandes marginales, il faut ajouter des interventions qui réduiront l'influence des pairs marginaux, sinon violents, par l'intégration d'adolescents qui affichent les signes précurseurs de la violence criminelle à des groupes d'adolescents prosociaux (voir par exemple le programme de Saint-Louis, exposé par Feldman, Caplinger et Wodarski, 1983). De plus, il faut penser à des programmes qui réduisent la probabilité de certains adolescents à risque de joindre une bande. Les programmes efficaces comportent les éléments suivants : douze heures d'information sur les bandes et des sujets connexes ainsi que des activités régulières après l'école : habiletés au travail, activités 
sportives et culturelles, aide scolaire et activités sociales (Brewer, Hawkins, Catalano et Neckerman, 1995).

Les cinq voies pour la prévention spécifique des comportements violents de certains groupes d'adolescents offrent toute une panoplie d'interventions qui influencent le niveau de violence dans les milieux où elles ont été mises en place. Toutefois, il existe un facteur qu'il ne faut pas négliger : les médias (Wilson et Herrnestein, 1985). En rapport avec ce phénomène social de la violence, ceux-ci jouent souvent, de façon involontaire, un rôle d'amplificateur. Il est nécessaire de parler de la violence interpersonnelle du point de vue de l'information de la population et du point de vue politique pour que le gouvernement affecte les ressources nécessaires à la prévention de la violence interpersonnelle. Par contre, la frontière entre traiter de la violence trop abondamment, et ainsi faire peur à la population, ou glorifier le phénomène et en parler de façon insuffisante est trop souvent difficile à tracer.

\section{Action sur les individus}

Les programmes de prévention de la délinquance sont amplement recensés par Tolan et Guerra (1994), Brewer et al. (1995), Tremblay et Craig (1995), Yoshikawa (1995) et Wasserman et Miller (1997). Compte tenu du thème de cet article, nous nous en tiendrons aux programmes qui ciblent explicitement les comportements violents et la violence criminelle. Cet inventaire des programmes de prévention et de traitement ne peut pas se limiter seulement à ceux qui ont fait l'objet d'une démonstration scientifique d'efficacité, ceci parce que, comme le soulignent avec à propos Guerra, Tolan et Hammond (1994), il y a très peu de programmes qui sont rigoureusement évalués et il y a encore moins de programmes avec une mesure de violence interpersonnelle comme critère de succès. En conséquence, nous mentionnerons les programmes rigoureusement évalués et occasionnellement des programmes dont le contenu semble s'appliquer particulièrement bien aux facteurs de risque des conduites violentes qui ont été analysées précédemment.

\section{La prévention primaire}

L'ensemble des programmes de prévention primaire de la violence veut réduire les comportements violents en améliorant les attitudes, les habiletés ou les comportements prosociaux des enfants et des adolescents. Ils s'adressent aux individus et ils ont généralement lieu à l'école. Les programmes qui sont offerts aux enfants ou aux adolescents sont en général d'une durée 
de dix à vingt sessions et ils comportent une ou plusieurs des composantes suivantes : la résolution de problèmes, la gestion du stress, l'estime et l'affirmation de soi, la sélection des pairs, l'autocontrôle, l'empathie, la résolution des conflits ou des informations diverses. Les programmes de cette nature, que recensent Guerra et al. (1994), rapportent des améliorations quant aux attitudes, habiletés ou comportements ciblés par ces composantes. Toutefois, les études ne démontrent pas le maintien à long terme d'aucun de ces acquis. Brewer et al. (1995) recensent d'autres programmes qui portent sur la gestion de la classe et l'organisation de l'école. Les conclusions de ces auteurs, comme les précédents, sont la faiblesse méthodologique de l'évaluation de ces programmes et le peu d'impact sur les comportements violents. Par exemple, le programme semblable de Prothrow-Stith (1991) rapporte une diminution marginale des bagarres autorapportées et des arrestations. Wasserman et Miller (1997) déplorent l'absence du critère de la violence pour les programmes recensés. Guerra et al. (1994) concluent leur recension en affirmant que ces programmes sont souvent trop généraux et de trop courte durée pour avoir un impact marqué et durable sur la violence. Ils suggèrent que ces programmes sont probablement plus efficaces pour encourager l'adhésion à des normes de non-violence, pour diminuer les opportunités de comportements violents et pour prévenir les comportements violents occasionnels des adolescents. Au Québec, Huot, Saint-Germain, Clark, Gauvin Fleurant (1996) proposent un programme d'information fort pertinent sur la violence, mais ce programme n'a pas encore été évalué et il a le désavantage de ne pas enseigner des habiletés pour faire face à la violence.

\section{La prévention secondaire}

La prévention secondaire concerne les personnes qui affichent déjà des comportements violents ou qui accumulent des facteurs de risque favorisant ces comportements. Cette forme de prévention exige un mécanisme de dépistage. Ces mécanismes varient énormément d'un programme à l'autre. Certains de ces mécanismes impliquent des instruments spécifiques, tandis que d'autres se limitent à un ou quelques facteurs de risque comme la monoparentalité ou la pauvreté (voir Le Blanc, 1997). Les programmes qui ciblent les individus choisissent deux catégories de personnes, les enfants agressifs ou les individus qui accumulent des facteurs de risque. Brewer et al. (1995) recensent les programmes de cette nature; ils notent que les programmes les plus intéressants et ceux dont 
l'efficacité est établie ciblent les habiletés individuelles et la famille, et que certains sont aussi de nature multimodale.

À titre d'exemple d'un programme qui cible les habiletés individuelles pour faire face à la tendance à opter pour la violence, il faut mentionner le programme " Positive Adolescents Choices Training » (PACT) qui comprend vingt heures d'entraînement à raison d'une heure par semaine et qui cible des adolescents qui vivent dans des communautés où la violence est endémique (voir Brewer et al., 1995). Les habiletés enseignées sont, entre autres, de donner de la rétroaction négative, de recevoir de la rétroaction négative et de négocier. Les participants à ce programme sont trois fois moins souvent référés au tribunal (17\% contre $47 \%$ ), et cela plus rarement pour des délits avec violence.

La famille fait l'objet de nombreuses actions de prévention. Deux types de programmes sont appliqués, il s'agit d'abord des interventions de support parental et ensuite de l'enseignement des méthodes disciplinaires (voir recension de Tremblay et Craig, 1995 ; Yoshikawa, 1995 ; Wasserman et Miller, 1997). Les premiers programmes identifient des mères à risque qui sont suivies directement dans leur milieu de vie après la naissance des enfants pour leur offrir tous les services nécessaires. Les seconds programmes de parentalité offrent aux parents d'enfants agressifs ou turbulents des activités d'apprentissage qui concernent la supervision et l'application de récompenses et sanctions appropriées. Parmi les programmes correctement évalués, beaucoup obtiennent une réduction des comportements marginaux et de la délinquance officielle selon les recensions (Tremblay et Craig, 1995 ; Yoshikawa, 1995 ; Wasserman et Miller, 1997), mais les évaluations ne démontrent pas une diminution réelle de l'agressivité ou de la délinquance avec violence.

Parmi les programmes multimodaux, l'intervention auprès des enfants agressifs de Montréal occupe une place à part par le contenu de l'intervention et par le fait qu'elle diminue les comportements agressifs chez les adolescents, comme la délinquance et d'autres comportements marginaux (Tremblay et al., 1991, 1992, 1994, 1997 ; Tremblay et Charlebois, 1988). Les enfants les plus agressifs en maternelle font l'objet d'une intervention de deux ans. Le programme comprend une composante parentalité (des rencontres environ aux trois semaines qui portent sur des lectures, l'entraînement à la supervision de leur fils, l'apprentissage des techniques de renforcement des comportements prosociaux, les méthodes pour punir efficacement sans abus et les manières de gérer les conflits familiaux, la gestion de l'écoute de la télévision, particulièrement des émissions 
violentes, et le support à la généralisation des apprentissages). Les enfants participent à un programme d'habiletés sociales à l'école. Ce programme comprend une dizaine de sessions chaque année (la première année les conduites prosociales sont ciblées tandis que la deuxième année les sessions portent sur l'autocontrôle). De plus, les professeurs de ces garçons agressifs reçoivent un support de l'équipe d'intervention quant à la gestion de leur classe et à leurs attitudes et comportements concernant l'enfant agressif. Finalement, des étudiants rencontrent, à plusieurs occasions chaque année, les enfants à la maison pour des activités de développement de leur vie imaginaire.

La prévention tertiaire ou les traitements les plus efficaces

Qu'il s'agisse des adolescents de la première ou de la seconde partie de l'adolescence ou des adultes au moment de la jeunesse, les marqueurs de la violence interpersonnelle renvoient à des interventions préventives relatives à l'intégration sociale, à la neutralisation de l'influence des pairs antisociaux et à leur fonctionnement psychosocial (Le Blanc, 1997). Mais puisque les comportements d'agression semblent persister dans le répertoire comportemental de l'individu, peut-être des interventions de réadaptation en profondeur sur les façons de penser et de réagir aux stimulations agressives ne seraient-elles pas seulement utiles mais aussi nécessaires. Différentes recensions et plusieurs méta-analyses ont été publiées sur l'efficacité des programmes de réadaptation pour les adolescents en difficulté (Gendreau et Ross, 1979 ; Gendreau et Andrew, 1990 ; Le Blanc, 1990b ; Lipsey, 1992 ; Palmer, 1994 ; Lipsey, 1997). D'autres recensions portent plus particulièrement sur les programmes de réadaptation qui essaient d'inclure une action spécifique sur la régulation de la colère et de l'agressivité (Feindler et Ecton, 1986 ; Goldstein et Glick, 1987 ; Gibbs, 1992 ; Goldstein, 1992 ; Hollin, 1992).

Lipsey (1997) a analysé 200 études évaluatives qui concernent des adolescents délinquants avec des antécédents criminels et dont les activités délinquantes sont sérieuses et souvent violentes. Il observe que les approches cognitives-comportementales ont davantage de succès, cela à la fois en milieu résidentiel et dans la communauté, tandis que l'approche individuelle, particulièrement la thérapie familiale multisystémique, est aussi efficace mais seulement dans la communauté. De l'ensemble de ces recensions et méta-analyses, certains programmes se démarquent pour l'intervention avec des adolescents violents qui provoquent le syndrome du « ce n'est pas un cas pour nous» («Dumping Syndrome», Agee, 1979). Ces 
programmes prometteurs prennent deux formes : la thérapie familiale multisystémique et l'approche cognitive-comportementale.

\section{Conclusion}

Nous avons analysé l'évolution récente de la violence interpersonnelle. Elle a progressé de la fin des années 1980 jusqu'au milieu des années 1990 et il semble que sa progression tende à diminuer. Nous avons soutenu que les facteurs macrosociaux n'étaient peut-être pas les plus importants pour expliquer cette évolution. Nous avons plutôt tenté de montrer que ce sont peut-être les modalités de socialisation des adolescents d'aujourd'hui qui sont plus responsables de l'accroissement récent de la violence interpersonnelle. Ces explications nous sont apparues plus pertinentes parce que les adolescents ne sont pas plus nombreux à manifester des agirs violents, mais ceux qui utilisent ces comportements le font avec une fréquence accrue. En s'appuyant sur ces données, nous avons inventorié les interventions préventives qui se sont avérées efficaces selon les études évaluatives. Le manque d'interventions évaluées rigoureusement est particulièrement flagrant au Québec.

Au terme de cet article, nous aimerions mentionner qu'il est peutêtre inapproprié de cibler seulement les comportements violents et les individus violents. Il est clairement établi, comme nous l'avons vu, que les conduites violentes s'insèrent dans l'ensemble du répertoire des conduites marginales des adolescents. En conséquence, les interventions de prévention primaire, secondaire ou tertiaire sont peut-être relativement peu efficaces parce que l'ensemble des conduites marginales, promiscuité sexuelle, consommation de psychotropes, rébellion familiale et scolaire, délinquance contre les biens, n'est pas considéré dans la plupart des interventions. Si les conduites marginales constituent un syndrome, comme beaucoup de travaux semblent le démontrer, une intervention sur la violence devient peut-être trop limitée.

\section{Références}

AgEe, V.L. (1979), Treatment of the Violent Incorrigible Adolescent, Lexington, Lexington Books.

Blumstein, A. (1995), "Youth Violence, Guns, and the Illicit-Drug Industry », Journal of Criminal Law and Criminology, vol. 86, p. 10-36. 
Bovasso, G., Jacobs, J. et RetTig, S. (1991), "Changes in Moral Values in Three Decades, 1958-1988 » Youth and Society, vol. 22, n 4, p. 468-481.

Brewer, D.D., Hawkins, J.D., Catalano, R.F. et Neckerman, H.J. (1995), «Preventing Serious, Violent, and Chronic Juvenile Offending : A Review of Selected Strategies in Childhood, Adolescence, and in the Community », in Howell, J.C., Krisberg, B., HaWkins, J.D., Wilson, J.J., Serious, Violent, and Chronic Juvenile Offenders, Thousand Oaks, Sage.

Brion-Meisels, S. et SELmAN, R.L. (1992), Fight, Flight or Collaboration? Individual and Institutional Development in the School, Working Paper, Faculty of Graduate Studies in Education, Harvard University, Cambridge (Mass).

Capaldi, D.M. et Patterson, G.R. (1996), «Can Violent Offenders Be Distinguished from Frequent Offenders: Prediction from Childhood to Adolescence ", Journal of Research in Crime and Delinquency, vol. 33, p. 206-231.

Chaiken, J., Chaiken, M. et Rhodes, W. (1994), « Predicting Violent Behavior and Classifying Violent Offenders », in REISS, A.J., ROTH, J.A., Understanding and Preventing Violence. Volume 4: Consequences and Control, Washington, National Academy Press.

Charlebois, P. (1992), «Behavioural and Cognitive Characteristics of Conduct Disordered-Hyperactive Boys from Age 6 to 11: A Multiple Informant Perspective », Journal of Child Psychology and Psychiatry, vol. 8, p. $1333-1346$.

Charlebois, P., Le Blanc, M., Gagnon, C. et Larivée, S. (1994), «Methodological Issues in Multiple-Gating Procedures for Antisocial Behaviors in Elementary Students », Remedial and Special Education, vol. 15, $\mathrm{n}^{\mathrm{o}} 1$, p. 44-55.

Conseil des affaires sociales (1989), Deux Québec dans un: rapport sur le développement social et démographique, Montréal, Gaëtan Morin, Gouvernement du Québec.

CoOK, P.J. et LAUB, J.H. (1996), «The Unprecedent Epidemic of Youth Violence ", Crime and Justice: An Annual Review, Chicago, University of Chicago Press.

Côté, G., Le Blanc, M., Ouellet, G. et Pronovost, A. (1988), Violence et délinquance à l'école : inventaire de mesures préventives, Montréal, Commission des écoles catholiques de Montréal, Service des études.

Cusson, M. (1990), Croissance et décroissance du crime, Paris, Presses universitaires de France.

Duguid, S. (1981), « The Prison As School », in AYERS, J.D. (dir.), Proceedings of the National Conference on Prison Education, Victoria, Canadian Association for Adult Education Correctional Services of Canada.

ElliotT, D.S. (1994), «Serious Violent Offenders : Onset, Developmental Course, and Termination — The American Society of Criminology 1993 Presidential Address », Criminology, vol. 32, p. 1-21. 
FARrington, D.P. (1989), «Early Predictors of Adolescent Aggression and Adult Violence », Violence and Victims, vol. 4, p. 79-100.

FARrington, D.P. (1991), « Childhood Aggression and Adult Violence ; Early Predictors and Later Life Outcomes », in Rubin, K.H. et PePler, D., The Development and Treatment of Childhood Aggression, Hilsdale, Lawrence Erlbaum.

FARRINGTON, D.P. (1997a), « Predictors, Causes, and Correlates of Male Youth Violence ", in TONry, M., Youth Violence Crime and Justice : An Annual Review, Chicago, University of Chicago Press.

FARRINGTON, D.P. (1997b), «Early Prediction of Violent and Non-Violent Youthful Offending ", European Journal on Criminal Policy and Research, vol. 5, $n^{\circ} 2$, p. 9-32.

FARrington, D.P. et LoEber, R. (1995), « Transatlantic Replicability of Risk Factors in the Development of Delinquency », Paper Given at the Meeting of the Society for Life History Research in Psychopathology in Chatham (Mass).

FeIndler, E.L. et ECTON, R.B. (1986), Adolescent Anger Control: Cognitivebehavioral Techniques, Elmsford, Pergamon.

Feldman, R.A., Caplinger, T.E. et Wodarsky, J.J. (1983), The Saint Louis Conundrum: The Effective Treatment of Antisocial Youth, Englewood Cliffs, Prentice Hall.

Felson, M. (1994), Crime and Everyday Life : Insight and Implications for Society, Thousands Oaks, Pine Forge Press.

Forgatch, M.S., Patterson, G.R. et Stootmiller, M. (1994), « Progressing Toward Violence : A Replication », Annual Meeting of the American Society of Criminology, Miami, November 1994.

FréChette, M. et Le Blanc, M. (1987), Délinquances et délinquants, Chicoutimi, Gaëtan Morin.

Gabor, T., Baril, M., Cusson, M., Élie, D., Le Blanc, M. et Normandeau, A. (1987), Armed Robbery : Cops, Robbers and Victims, Springfield, Charles C. Thomas.

Gendreau, G. (1978), L'intervention psycho-éducative: solution ou défi?, Paris, Fleurus.

Gendreau, G. (1991), « Pour bien prendre le virage de l'an 2000 : les défis de l'action psychoéducative », Revue canadienne de psycho-éducation, vol. 20, $\mathrm{n}^{\circ} 1$, p. 29-39.

Gendreau, P. et Andrews, D.A. (1990), «What the Meta-Analyses of the Offender Treatment Literature Tells Us About "What Works" », Canadian Journal of Criminology, vol. 32, p. 173-184.

Gendreau, P. et Ross, R.R. (1979), «Effective Correctional Treatment: Bibliotherapy for Cynics », Crime and Delinquency, vol. 25, n 4, p. 463-489. 
GiBBS, J.C. (1992), « Moral-Cognitive Interventions », in GoldSTEIN, A.P. et HufF, C.R. (dir.), The Gang Intervention Handbook, Champaign, Research Press.

Gibis, J.C., Potter, G.B. et Goldstein, A.P. (1995), The EQUIP Program: Teaching Youth to Think and Act Responsibly Through a Peer-Helping Approach, Champaing, Research Press.

GIRARD, S. (1995), L'activité déviante, phénomène unidimensionnel ou multidimensionnel: une comparaison temporelle des pupilles de la Chambre de la jeunesse de Montréal de 1974 et de 1992, mémoire de maîtrise inédit, École de criminologie, Université de Montréal.

Gold, M. et ReImer, D.J. (1975), « Changing Patterns of Delinquent Behavior Among Americans 13 through 16 Years Old: 1967-72», Crime and Delinquency, vol. 7, $\mathrm{n}^{\circ} 4$, p. 483-517.

Gold, M. et OsGood, D.W. (1992), Personality and Peer Influence in Juvenile Corrections, Westport, Greenwood Press.

Goldstein, A.P. (1988), The Prepare Curriculum : Teaching Prosocial Competencies, Champaign, Research Press.

GoldsteIN, A.P. (1992), «Interpersonal Skills Training Interventions », in Goldstein, A.P. et Huff, C.R. (dir.), The Gang Intervention Handbook, Champaign, Research Press.

Goldstein, A.P. et Glick, B. (1987), Aggression Replacement Training: A Comprehensive Intervention for Aggressive Youth, Research Press.

Goldstein, A.P., Glick, B., Carthan, W. et Blancero, D.A. (1994), The Prosocial Gang : Implementing Aggression Replacement Training, Thousands Oaks, Sage.

Goldstein, A.P., Glick, B., Irwin, M.-J., Past-McCartney, C. et Rubama, I. (1989), Reducing Delinquency, New York, Pergamon Press.

GotTfredson, D.C. (1986), «An Empirical Test of School Based Environmental and Individual Interventions to Reduce their Risk of Delinquent Behavior », Criminology, vol. 24, p. 705-731.

GotTFredson, D.C. (1987), « An Evaluation of an Organization Development Approach to Reducing School Disorder », Evaluation Review, vol. 11, p. 739763.

Gottfredson, D.C., Karweit, N.L. et GotTfredson, G.D. (1989), Reducing Disordely Behavior in Middle Schools, Baltimore, John Hopkins University, Center for Research on Elementary and Middle Schools.

Gottrredson, D.C., Gottfredson, G.D. et Hybl, L.G. (1993), « Managing Adolescent Behavior : A Multiyear Multischool Study », American Educational Research Journal, vol. 30, p. 179-215.

Gottfredson, D.C., Gottrredson, G.D., Skrokan, S. (1996), «A Multimodel School Based Prevention Demonstration », Journal of Adolescent Research, vol. 11, p. 97-115. 
Greenwood, P.W., Model, K.E., Rydell, C.P. et ChIESA, J. (1996), Diverting Children from a Life of Crime: What Are the Cost and Benefits?, Santa Monica, Rand.

Guerra, N.G., Tolan, P.H. et Hammond, R. (1994), «Prevention and Treatment of Adolescent Violence ", in REISS, A.J. et ROTH, J.A., Understanding and Preventing Violence. Volume 4: Consequences and Control, Washington, National Academy Press.

HAMPARIAM, D.M., Davis, J.M., JACOBSON, J.M. et MCGraW, R.E. (1985), The Young Criminal Years of the Violent Few, Washington, National Institute for Juvenile Justice and Delinquency Prevention.

Hampariam, D.M., Shuster, R., Dinitz, S. et ConRad, J.P. (1978), The Violent Few, A Study of Dangerous Juvenile Offenders, Toronto, Lexington.

Hawkins, J.D., Catalano, R.F. et Brewer, D.D. (1995), « Preventing Serious, Violent, and Chronic Offending : Effective Strategies from Conception to Age 6 », in Howell, J.C., Krisberg, B., Hawkins, J.D. et Wilson, J.J., Serious, Violent, and Chronic Juvenile Offenders, Thousand Oaks, Sage.

Hawkins, J.D., Herrenkohl, T., Farrington, D.P., Brewer, D. et Catalano, R.F. (1997), « A Review of Predictors of Youth Violence », in LOEBER, R. et FARRINGTON, D.P., Serious and Violent Juvenile Offenders: Risk Factors and Successful Interventions, Washington, Office of Juvenile Justice and Delinquency Prevention Study Group on Serious / Violent / Chronic Offenders, Beverly Hills, Sage.

HÉBERT, J. (1989), «La problématique des jeunes agressifs : des points de repère », Apprentissage et socialisation en piste, vol. 12, $\mathrm{n}^{\circ} 1$, p. 45-52.

Henggeler, S.W. et Borduin, C.M. (1990), Family Therapy and Beyond: A Multisystemic Approach to Treating the Behavior Problems of Children and Adolescents, Pacific Grove, Brooks / Cole.

Henggeler, S.W., Cunningham, P.B., Pickrel, S.G., Schoebwaild, S.K. et Brondino, M.J. (1996), «Multisystemic Therapy: An Effective Violence Prevention Approach for Serious Juvenile Offenders », Journal of Adolecence, vol. 19 , p. $47-61$.

Henggeler, S.W., Mellton, G.B., Smith, L.A., Schoenwald, S.K. et Hanley, J.H. (1993), « Family Preservation Using Multisystemic Treatment : A LongTerm Follow-Up to a Clinical Trial with Serious Juvenile Offenders », Journal of Child and Family Studies, vol. 2, p. 283-293.

Henggeler, S.W., Schoenwald, S.K., Brondino, M.J., Cinningham, P.B., DAS, S., et al. (1995), Multisystemic Therapy Using Home-Based Services: A Clinically Effective and Cost Effective Strategy for Treating Serious Clinical Problems in Youth, Charleston, Family Services Research Center, Department of Psychiatry and Behavioral Sciences, Medical University of South Carolina.

Hickey, J. et Scharf, P. (1980), Toward a Just Correctional System, San Francisco, Jossey-Bass. 
HoGH, E. et Wolf, P. (1983), « Violent Crime in a Birth Cohort : Copenhagen 1953-1977 », in Van Deusen, K.T. et MednicK, S.A., Prospective Studies in Crime and Delinquency, Boston, Kluwer-Nijhoff.

Hollin, C.R. (1990), Cognitive-Behavioral Interventions with Young Offenders, Elmsford, Pergamon.

Huesman, L.R., Eron, L.D., LefKowitz, M.M. et Walder, L.O. (1984), «Stability of Aggression Over Time and Generations», Developmental Psychology, vol. 20, n 6, p. 1120-1134.

Huot, R., St-Germain, Clark D. et Gauvin Fleurant, A. (1996), Briser les chaînes, Ville Lemoyne, Programme régional d'intervention masculine AprèsCoup.

Jennings, W.S. et KoHlberG, L. (1983), « Effects of Just Community Programs on the Moral Development of Youthful Offenders », Journal of Moral Education, vol. 12, p. 33-55.

KASPY, N. (1995), Construction of Deviant Behavior Classification Systems for Boys Ages 12 to 18, mémoire de maitrise inédit, École de criminologie, Université de Montréal.

LANCTÔT, N. et LE BlANC, M. (1996), «La participation des garçons à une bande marginale: un phénomène de sélection et d'opportunités », Revue canadienne de criminologie, vol. 38, p. 375-400.

LANGELIER-Biron, L. (1989), « La violence dans le Québec des années 1980 », Apprentissage et socialisation en piste, vol. 12, $\mathrm{n}^{\circ} 1$, p. 37-44.

Le Blanc, M. (1983), Boscoville: la rééducation évaluée, Montréal, Éditions Hurtubise $\mathrm{HMH}$.

LE BLANC, M. (1990a), « Le cycle de la violence physique : trajectoire sociale et cheminement personnel de la violence individuelle et de groupe », Criminologie, vol. 23, $\mathrm{n}^{\circ} 1$, p. 47-74.

LE BLANC, M. (1990b), «L'intervenant auprès des jeunes délinquants, omnipraticien ou spécialiste », Revue canadienne de psycho-éducation, vol. 19, $\mathrm{n}^{\circ}$ 2, p. 85-100.

LE BLANC, M. (1991), «Violences, bandes, minorités ethniques et réadaptation des jeunes délinquants », Revue canadienne de psycho-éducation, vol. 20, p. 139150.

Le Blanc, M. (1995), «Common, Temporary, and Chronic Delinquencies : Prevention Strategies During Compulsory School », in WiKströM, P.-O., MCCORD, J. et ClARKE, R.W., Integrating Crime Prevention Strategies : Motivation and Opportunity, Stockholm, The National Council for Crime Prevention, p. 169-205.

Le Blanc, M. (1996a), "Changing Patterns in the Perpetration of Offences Over Time : Trajectories from Onset to the Middle of the Thirties », Studies on Crime and Crime Prevention, vol. 5, p. 151-165 
LE BLANC, M. (1996b), MASPAQ mesures de l'adaptation sociale et personnelle pour les adolescents québécois : manuel, Montréal, École de psycho-éducation, Groupe de recherche sur les adolescents en difficulté, Université de Montréal.

LE BlanC, M. (1997), «Screening Serious and Violent Juvenile Offenders : Identification, Classification, and Prediction ", in LOEBER, R. et FARRINGTON, D.P., Serious and Violent Juvenile Offenders: Risk Factors and Successful Interventions, Washington, Office of Juvenile Justice and Delinquency Prevention Study Group on Serious / Violent / Chronic Offenders, Beverly Hills, Sage.

Le BlanC, M. et CôtÉ, G. (1986), « Comparaison des adolescents de 14-15 ans en 1974 et en 1985 », in Tremblay, R.E., Le BlanC, M. et SChWARTZMan, A.E., La conduite délinquante des adolescents de Montréal (1974-1985) : étude descriptive et prédictive, Montréal, École de psycho-éducation, Université de Montréal.

Le Blanc, M., Dionne, J., ProulX, J. et Grégoire, J. (1997), Intervenir autrement : apparier adolescents en difficulté et méthodes d'intervention. Qui? Quoi? Comment?, Montréal, Presses de l'Université de Montréal.

Le Blanc, M. et Fréchette, M. (1989), Male Criminal Activity from Childhood through Youth : Multilevel and Developmental Perspectives, New York, SpringerVerlag.

LE BlanC, M. et GiRARD, S. (1997), « The Generality of Deviance : Replication Over Several Decades with a Canadian Sample of Adjudicated Boys », Canadian Journal of Criminology, vol. 39, $\mathrm{n}^{\circ}$ 2, p. 171-183.

Le Blanc, M., Girard, S., Kaspi, N., Lanctôt, N. et Langelier, S. (1995), Les adolescents en difficulté des années 1990, rapport $n^{0} 3$. Adolescents protégés et jeunes contrevenants sous ordonnance de la Chambre de la jeunesse de Montréal en 1992 1993, Montréal, Groupe de recherche sur les adolescents en difficulté, Université de Montréal.

Le Blanc, M., HéBert, C. et David, P. (1988), Prédiction de l'inadaptation à l'âge adulte, Centre international de criminologie comparée, Université de Montréal.

Le Blanc, M., Janosz, M. et Langelier-Biron, L. (1993), «L'abandon scolaire : antécédents sociaux et personnels et prévention spécifique ", Apprentissage et socialisation, vol. $16, \mathrm{n}^{\circ} 1-2$, p. 43-64.

LE BLANC, M. et KASPY, N. (sous presse), «Trajectories of Delinquency and Problem Behavior: Comparison of Synchronous and Non Synchronous Paths on Social and Personal Control Characteristics of Adolescent », Journal of Quantitative Criminology.

LE BlanC, M. et LANCTÔT, N. (1995), « Le phénomène des bandes marginales, vers une vision réaliste grâce à une comparaison des années 1970 et 1990 », Revue internationale de criminologie et de police technique, vol. $95, \mathrm{n}^{\circ} 4$, p. 414426. 
LE Blanc, M. et LANCTÔT, N. (1997), La participation à une bande marginale : continuité et conséquences, Montréal, Groupe de recherche sur les adolescents en difficulté, École de psychoéducation, Université de Montréal.

Le Blanc, M., Ouimet, M., et Tremblay, R.E. (1988), « An Integrated Theory of Delinquent Behavior, 1976-1985 », Psychiatry, vol. 51, n 2, p. 164-176.

Le Blanc, M. et Pronovost, L., (1979), « Le passage de l'école au travail et la délinquance », Apprentissage et socialisation, vol. 2, n ${ }^{\circ} 2$, p. 69-74.

Le Blanc, M. et Pronovost, L., (1980), « Transition statutaire et délinquance », Revue canadienne de criminologie, vol. 22, p. 288-297.

Le Blanc, M. et Tremblay, R.E. (1988), « Homeostasis : Social Changes Plus Modifications in the Basic Personality of Adolescents Equal Stability of Hidden Delinquency », International Journal of Adolescence and Youth, vol. 1, $\mathrm{n}^{\circ}$ 3, p. 269-291.

Le Blanc, M., Vallières, E., et McDuff, P. (1992), «Adolescents' School Experience and Self-Reported Offending, a Longitudinal Test of a Social Control Theory », International Journal of Adolescence and Youth, vol. 3, $\mathrm{n}^{\mathrm{os}} 3$ 4, p. 197-247.

Le Blanc, M., Vallières, E., et McDuff, P. (1993), « The Prediction of Males Adolescent and Adult Offending from School Experience », Canadian Journal of Criminology, vol. 35, $\mathrm{n}^{\circ}$ 4, p. 459-478.

LIPSEY, M.W. (1989), Juvenile Delinquency Treatment : A Meta-Analysis Inquiry into the Variablility Effects, New York, Russel Sage Foundation.

LIPSEY, M.W. (1992), «Juvenile Delinquency Treatment: A Meta-Analytic Inquiry into the Variability of Effects ", in COOK, T.D., COOPER, H., Cordray, D.S., Hartman, H., Hedges, L.V., Light, R.J., Lovis T.A. et Mosteller, S.M. (dir.), Meta-Analysis for Explanation : A Casebook, New York, Sage.

Lipsey, M. et WilsON, M. (1997), «Effective Intervention of Serious Juvenile Ofenders : A Synthesis of Research », in Loeber, R. et FARrington, D. (dir), Serious / Violent / Chronic Offenders, Washington, Office of Juvenile Justice and Delinquency Prevention Study Group on Serious / Violent / Chronic Offenders.

Loeber, R. (1985), «Patterns and Development of Antisocial Child Behavior », in WhitehURST G.J., Annals of Child Development, vol. 2, p. 138166.

LOeBER, R. et HAY, D. (1997), « Key Issues in the Development of Aggression and Violence from Childhood to Early Adulthood», Annual Review in Psychology, vol. 48, p. 371-410.

Loeber, R., Keenan, K. et Zhang, Q. (1997), « Boys' Experimentation and Persistance in Developmental Pathways Toward Serious Delinquency », Journal of Children and Family Studies, in Press. 
Loeber, R. et LE BLANC, M. (1990), « Toward a Developmental Criminology », in TONry, M. et Morris N., Crime and Justice : An Annual Review, vol. 13, p. 198, Chicago, University of Chicago Press.

Loeber, R. et Le Blanc, M. (1997), «An Update On Developmental Criminology: The Development of Juvenile Offending in the Context of Deviant Behavior ", in TONRY, M., Crime and Justice : An Annual Review, vol. 20, Chicago, University of Chicago Press.

Loeber, R., Smalley, M.M., KeEnAN, K. et Zhang, Q. (1997), « A Prospective Replication of Developmental Pathways in Disruptive and Delinquent Behavior », in CAIRNS, R.B., The Individual as a Focus in Developmental Research, Beverly Hills, Sage.

Loeber, R. et Southamer-Loeber, M. (1987), "Prediction », in QuAY, H.C., Handbook of Juvenile Delinquency, New York, John Wiley \& Sons.

Loeber, R., Wung, P., Keenan, K., Giroux, B., Stiuthamer-Loeber, M. et Van Kammen, W.B. (1993), « Developmental Pathways in Disruptive Child Behavior », Developmental Psychopathology, vol. 5, p. 101-132.

Miller, S.J., Dinitz, S. et CONRAD, J.P. (1982), Careers of the Violent: the Dangerous Offender and Criminal Justice, Lexington, Lexington Books.

Novaco, R.W. (1975), Anger Control: The Development and Evaluation of an Experimental Treatment, Lexington, Heath.

Olweus, D. (1979), «Stability of Aggressive Reaction Patterns in Male: A Review », Psychological Bulletin, vol. 86, p. 852-875.

Olweus, D. (1991), « Bully / Victim Problems Among School Children : Basic Facts and Effects of a School-Based Intervention Program », in RUBIN, K.H. et Pepler, D., The Development and Treatment of Childhood Aggression, Hilsdale, Lawrence Erlbaum.

OsGood, D.W., O’Mallley, P.M., BAChMAN, J.G. et JOHNSTON, L.D. (1989), «Time Trends and Age Trends in Arrests and Self-Reported Illegal Behavior ", Criminology, vol. 27, n 3, p. 389-417

Ouimet, M., Tremblay, P. et Morselli, C. (1997), « Analyse stratégique des facteurs démographiques, économiques et sociaux qui façonnent l'environnement du Service de police de la Communauté urbaine de Montréal », Montréal, Centre international de criminologie comparée, Université de Montréal.

Palmer, T. (1994), A Profile of Correctional Effectiveness and New Directions for Research, Albany, State University of New York Press.

PANCOAST, D.L. et ARCHER, R.P. (1988), « MMPI Adolescent Norms : Patterns and Trends Across 4 Decades ", Journal of Personality Assesment, vol. 52, $\mathrm{n}^{\circ} 4$, p. 691-706.

Prothrow-Stith, D. (1991), Deadly Consequences : How Violence is Destroying our Teenage Population and a Plan to Begin Solving the Problem, New York, Harper Collins. 
ROFF, M. et WIRT, R.D. (1984), « Childhood Aggression and Social Adjustment as Antecedents of Delinquency », Journal of Abnormal Child Psychology, vol. 12, p. 11-26.

RoY, J. (1989), «L'inévitable violence », Apprentissage et socialisation en piste, vol. $12, n^{\circ} 1$, p. 29-36.

Sebald, H. (1989), « Adolescents' Peer Orientation : Changes in the Support System During the Past Three Decades », Adolescence, vol. 24, nº 96, p. 937 946.

Slaby, R. et Guerra, N. (1990), "Cognitive Mediators of Aggression in Adolescent Offenders : Intervention », Developmental Psychology, vol. 26, $\mathrm{n}^{\circ}$ 2, p. 269-277.

SNyder, H.N., Sickmund, M. et Poe-Yamagata, E. (1996), Juvenile Offenders and Victims : 1996 Update on Violence, Washington, Office of Juvenile Justice and Delinquency Prevention, U.S. Department of Justice.

Spergel, I.A. (1995), The Youth Gang Problem. A Community Approach, New York, Oxford University Press.

Thornberry, T.P., Krohn, M.D., Lizotte, A.J. et Chard-Wierschem, D. (1993), « The Role of Juvenile Gangs in Facilitating Delinquent Behavior », Journal of Research in Crime and Delinquency, vol. 30, p. 55-87.

THORnBerry, T.P. (1997), « Membership in Youth Gangs and Involvement in Serious and Violent Offending », in Loeber, R. et FARRINGTON, D. (dir.), Serious / Violent / Chronic Offenders, Washington, Office of Juvenile Justice and Delinquency Prevention Study Group on Serious / Violent / Chronic Offenders.

Tolan, P.H. et Guerra, N.G. (1994), « Prevention of delinquency : Current Status and Issues », Applied and Predictive Psychology, vol. 3, p. 251-273.

Tremblay, R.E. et Charlebois, P. (1988), La prévention du développement de comportements antisociaux chez les jeunes garçons agressifs, effets observés à la fin de l'intervention, Montréal, Groupe de recherche sur l'inadaptation psychosociale chez l'enfant, Université de Montréal.

Tremblay, R.E. et Craig, W.M. (1995), « Developmental Crime Prevention », in TONRY, M. et FARrington, D.P., Building a Safer Society: Strategic Approaches to Crime Prevention, Crime and Justice: An Annual Review, vol. 19, p. 151-236, Chicago, University of Chicago Press.

Tremblay, R.E. et Desmarais-Gervais, L. (1986), «La prédiction de l'inadaptation de l'enfance à l'adolescence », in TREMBLAY, R.E., LE BLANC, M. et Schwatzman, A.E., La conduite délinquante des adolescents à Montréal (1974-1985) ; étude descriptive et prédictive, Montréal, Université de Montréal.

Tremblay, R.E., Kurtz, L.C., Masse, F., Vitaro, F. et Phil, R.O. (1994), $A$ Bimodal Preventive Intervention for Disruptive Kindergarden Boys : Its Impact Through Mid-Adolescence, Unpublished manuscript, Montreal, Research Unit on Children's Psychosocial Maladjustment, Université de Montréal. 
Tremblay, R.E., Le Blanc, M. et Schwartzman, A. (1988), « The Predictive Power of First Grade and Teacher Rating: Sex Differences in Antisocial Behavior and Personnality at Adolescence ", Journal of Abnormal Child Psychology, vol. 16, n⿳3 3, p. 571-583.

Tremblay, R.E., Loeber, R., Gagnon, C., Charlebois, P., Larivée, S. et Le BlanC, M. (1991), «Disruptive Boys with Stable and Unstable Fighting Behavior Patterns During Junior Elementary School », Journal of Abnormal Child Psychology, vol. 19, nº 3, p. 285-300.

Tremblay, R.E., Masse, L.C., Kurtz L. et Vitaro, F. (1997), «From Childhood Physical Aggression to Adolescent Maladjustment : the Montréal Prevention Experiment », in Peters, R.D. et MCMahon, R.J., Childhood Disorders, Substance Abuse, and Delinquency: Prevention and Early Intervention Approaches, Thousands Oaks, Sage.

Tremblay, R.E., McCord, J., Boileau, H., Charlebois, P., Gagnon, C., Le Blanc, M. et Larivée, S. (1991), "Can Disruptive Boys Be Helped to Become Competent? », Psychiatry, vol. 54, p. 148-161.

Tremblay, R.E., Vitaro, F., Le Blanc, M., Bertrand, L., Beauchesne, H., Boileau, H. et David, L., (1992), « Parent and Child Training to Prevent Early Onset of Delinquency: the Montréal Longitudinal-Experimental Study » in MCCORD, J. et Tremblay, R.E. (dir.), Preventing Deviant Behavior from Birth to Adolescence, New York, Guilford Press.

Vitaro, F., Dobkin, P.L., Gagnon, C. et Le BlanC, M. (1994), Les problèmes d'adaptation psychosociale chez l'enfant et l'adolescent : prévalence, déterminants et prévention, Montréal, Presses de l'Université du Québec.

WASSERman, G. et Miller, L. (1997), «The Prevention of Antisocial Behavior », in LOEBER, R. et FARrington, D. (dir.). Serious / Violent / Chronic Offenders, Washington, Office of Juvenile Justice and Delinquency Prevention Study Group on Serious / Violent / Chronic Offenders.

WiKSTRÖM, P.-O.H. (1985), Everyday Violence in Contemporary Sweden : Situational and Ecological Approach, Stockholm, The National Council for Crime Prevention, Report $\mathrm{n}^{\circ} 15$.

WiKström, P.-O.H. (1987), Patterns of Crime in a Birth Cobort, Stockholm, University of Stockholm, Department of Sociology.

Wilson, J.Q. et Herrnestein, R.J. (1985), Crime and Human Nature, New York, Simon \& Schuster.

Wolfgang, M.E., Thornberry, T.P. et Figlio, R.M. (1987), From Boy to Man, from Delinquency to Crime, Chicago, University of Chicago Press.

YoshiKawa, H. (1995), « Long-term Effects of Early Childhood Programs on Social Outcome and Delinquency », The Future of Children, vol. 5, $\mathrm{n}^{\circ} 3$. 


\section{Criminologie}

Revue semi-thématique semestrielle

Directeur : Pierre Landreville

Criminologie, revue de recherche qui s'adresse aux scientifique et aux professionnels de la justice pénale, présente des dossiers thématiques construits autour des préoccupations et des intérêts actuels des criminologues québécois, canadiens, américains et européens. Chaque article est accompagné d'un résumé en anglais. et en français

Déjà parus :

$\square$ La sécurité privée $15,00 \$$

$\square$ La prévention de l'inadaptation sociale :

approche développementale $15,00 \$$

$\square$ Violences familiales $15,00 \$$

$\square$ Criminalités économiques $15,00 \$$

L'homicide conjugal au Canada 15,00\$

Intervenir auprès des délinquants $15,00 \$$

$\square$ La détention provisoire $15,00 \$$

$\square$ L'histoire de l'enfermement et des

populations pénales $11,00 \$$

$\square$ Sexe et criminalité $10,50 \$$

$\square$ Analyse spatiale du crime $10,50 \$$

$\checkmark$ La criminologie comparée

Hommage à Denis Szabo 9, 25 \$

$\square$ Michel Foucault et la (post)modernité 12, $50 \$$

$\checkmark$ Nouvelles connaissances et nouvelles questions en criminologie $9,00 \$$

$\square$ Les femmes et le contrôle pénal 9,00\$

$\square$ Regard sur les tribunaux $8,50 \$$

$\square$ Les droits des détenu-e-s $8,50 \$$
Après le crime : survivre $8,00 \$$

Nouvelles violences à l'adolescence $8,00 \$$

$\square$ Le milieu criminel 7, $50 \$$

Drogues et criminalités 7, $50 \$$

Les soins de santé mentale en milieu carcéral 7, 50 \$

$\square$ Entre les mailles de la loi 7, 50 \$

La détermination de la sentence $7,50 \$$

Faire les nouvelles. Journalisme

et affaires criminelles 7, $50 \$$

La prédiction de la carrière criminelle $7,50 \$$

$\checkmark$ Politiques et pratiques pénales. 25 ans

de réflexion et d'action $15,00 \$$

$\square$ Le vol à main armée à Montréal 7,50 \$

$\square$ Histoire du contrôle social 7, 50 \$

$\square$ La dangerosité $7,50 \$$

$\square$ La police après $19847,50 \$$

Les femmes et la justice pénale 7, 50 \$

$\square$ La peur du crime 7, $50 \$$

$\square$ Droit et justice 7, 50 \$

$\square$ Libération conditionnelle. Évolution

et application (1899-1981) 7, 50 \$
Service d'abonnements

FAXON QUÉBEC

C.P 48884, Outremont (QC)

Canada H2V 4V3

Tél. : (514) 274-5468

1-800-361-1431

Téléc. : (514) 274-0201

Dépositaire Europe

LIBRAIRIE DU QUÉBEC

30, rue Gay-Lussac

75005 Paris (France)

Tél. : 1.43.54.49.02

Téléc. :1.43.54.39.15

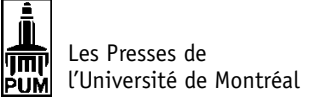

C.P. 6128, succ. Centre-Ville

Montréal (Québec)

$\mathrm{H} 3 \mathrm{C} 3 \mathrm{~J} 7$

Tél. : (514) 343-6933

Télec. : (514) 343-2232

Courriel : pum@umontreal.ca

\section{Bulletin d'abonnement}

$\square$ Je désire m'abonner à Criminologie pour l'année

$\square$ Veuillez m'expédier les titres cochés

$\square$ Paiement ci-joint (chèque ou mandat) à l'adresse postale Plus 7 \% TPS (non applicable à l'extérieur du Canada)

$\square$ Visa

- Master Card

$\mathrm{N}^{\circ}$

Date d'expiration

Nom

Adresse

Code postal

Abonnement annuel 1999 (Volume 32)

Individus :

Canada

$27,00 \$$

Pays étrangers 32. $00 \$$ US

Étudiant avec $\mathrm{n}^{\circ}$ de carte $\quad 20,00 \$$

Institutions :

Canada 53, $00 \$$

Étranger 53. $00 \$$ US 


\section{REVUE CRIMINOLOGIE CONSIGNES AUX AUTEURS}

Criminologie ne publie que des textes originaux et inédits en français sur des résultats de recherche se rapportant à la délinquance, à la criminalité et à leur contrôle. Les articles sont évalués anonymement par un comité de lecture extérieur au comité de rédaction.

Tout article ne respectant pas les normes ci-dessous énumérées sera retourné à son auteur.

\section{SOUMISSION DES TEXTES}

Les textes doivent parvenir à la rédaction en quatre (4) exemplaires, accompagnés d'une disquette sur laquelle le logiciel et la plate-forme utilisés sont précisés. Ex: Word 97 pour Windows. Dans la mesure du possible, nous faire parvenir le texte dans une version récente du logiciel Microsoft Word.

Joindre les fichiers de toutes polices de caractères particulières, que ce soit pour les documents textuels ou les fichiers images (eps).

\section{Présentation des manuscrits}

Les textes doivent être présentés sur format papier, à double interligne. Ils ne doivent pas contenir plus de 6000 mots.

La première page ne doit présenter que le titre du texte.

Sur la deuxième page doit apparaître le nom des auteurs, leur affiliation professionnelle, leur courriel, ainsi que le nombre de mots.

Deux résumés d'au plus 15 lignes chacun, l'un en anglais et l'autre en français, doivent être présentés sur la troisième page. Parce que les textes sont évalués à l'aveugle, il est très important que les auteurs s'assurent que les articles soumis ne contiennent aucune information permettant de les identifier.

TABLEAUX

Les tableaux doivent être identifiés dans un ordre séquentiel selon le modèle suivant : Tableau 1, Tableau 2 etc.

Les tableaux doivent être créés avec les fonctionnalités appropriées du traitement de texte. Les items des cellules ne devraient donc pas être séparés avec des tabulations mais plutôt avec de véritables cellules.

Les tableaux doivent être insérés dans le texte après le premier paragraphe où on y réfere pour la première fois.

\section{FIGURES}

Les figures doivent être identifiées dans un ordre séquentiel selon le modèle suivant : Figure 1, Figure 2, etc.

Joindre des originaux imprimés et des versions électroniques des figures de préférence, en formats TIFF, EPS ou Photoshop avec une excellente résolution.

\section{NOTES}

Les notes doivent être numérotées et apparaître en bas de page. Utiliser la fonction appropriée du traitement de texte pour lier les notes de bas de page avec les appels de notes dans le texte.

Dans le texte, les appels de notes sont en exposant et se trouvent immédiatement après le passage auquel ils renvoient et avant la ponctuation.

Les auteurs doivent éviter d'utiliser les notes pour expliquer et approfondir leurs propos et les réserver pour des précisions essentielles. 


\section{RÉFÉRENCES CITÉES dANS LE TEXTE}

Ne pas citer les renseignements bibliographiques en entier dans le texte ou en notes infrapaginales. Il suffit d'indiquer, entre parenthèses, le nom de l'auteur suivi de l'année de publication. S'il y a lieu, indiquer les pages auxquelles on se réfere en les faisant précéder d'un deux-points. Ex : (Boileau, 1991 : 312-313).

Si le nom de l'auteur est déjà mentionné dans le texte, le faire suivre par l'année (et les pages s'il y a lieu) entre parenthèses. Ex : Boileau (1991).

Lorsqu'un auteur a plus d'un ouvrage publié la même année, les distinguer par les lettres a, b, c, etc., ajoutées à l'année. Ex : (Boileau, 1991a).

Si plusieurs auteurs sont mentionnés, les indiquer par ordre croissant d'année de publication et les séparer par un point-virgule. Ex : (Fagnan, 1991; Dupuis, 1995; Tardif, 1998).

Si un ouvrage compte deux auteurs, mentionner les deux noms.

Si un ouvrage compte plus de deux auteurs, ne mentionner que le premier nom suivi de « et al. » en italique. Ex : (Bourbonnais et al., 1997).

Dans le cas d'un organisme, mentionner le nom au complet lors de la première citation. Il est possible d'utiliser une abréviation, un sigle ou un acronyme par la suite, en autant que ce dernier ait été mentionné lors de la première citation. Ex : (Office national du film du Canada (ONF), 1992).

Les références complètes devront apparaître dans la liste des références, à la fin du texte.

\section{LISTE DES RÉFÉRENCES}

La liste des références doit être présentée à la fin du texte dans une section intitulée « Références ».

Lorsque plusieurs références se rapportent à un même auteur, les présenter en ordre croissant d'année de publication.

Lorsqu'une référence comporte plusieurs auteurs, tous les noms doivent être mentionnés. Ne pas utiliser et al. dans la liste des références.

\section{NORMES À SUIVRE POUR LA PRÉSENTATION DES RÉFÉRENCES}

Les noms des auteurs sont saisis en petites capitales.

Livre :

SANDERS, D.H., MURPH, A.F. et ENG, R.J. 1984. Les statistiques, une approche nouvelle, Montréal : McGraw-Hill Éditeurs.

\section{Article :}

BRILLON, Y. 1986. «L'opinion publique et les politiques criminelles », Criminologie 19 (1) : 227-238.

\section{Chapitre d'un livre :}

LASVERGNAS, I. 1987. «La théorie et la compréhension du social », p.111-173 in Recherche sociale, sous la direction de B. Gauthier. Sillery : Presses de l'Université du Québec. 


\section{CRIMINOLOGIE OUVRE SES PAGES}

Avez-vous récemment réalisé un projet de recherche touchant un aspect de la criminologie ou de la justice pénale? Aimeriez-vous que vos travaux soient connus d'un vaste public?

Dans chacun de ses numéros, la revue Criminologie publie des textes de recherche présentant divers aspects d'une même problématique, sous forme d'un DOSSIER thématique. Toutefois, chaque numéro réserve également une section aux articles HORS THÈME. Cette section regroupe les articles soumis par des chercheurs et des chercheuses qui désirent rendre compte des résultats de leurs recherches récentes. Nous sommes désireux de vous faire connaître cette section tout en vous rappelant qu'elle offre des possibilités pour la publication de vos résultats de recherche.

Pour soumettre vos articles n'excédant pas 6000 mots ou obtenir de plus amples renseignements, veuillez vous adresser au :

Secrétariat de rédaction Revue Criminologie Centre international de criminologie comparée Université de Montréal

C.P. 6128, succ. Centre-Ville Montréal (Québec), Canada $\mathrm{H} 3 \mathrm{C} \quad 3 \mathrm{~J} 7$

Sylvie Gravel : (514) 343-2120 sylvie.gravel@umontreal.ca Télécopieur : (514) 343-2269 